\title{
Description of self-synchronization effects in distributed Josephson junction arrays using harmonic analysis and power balance
}

\author{
J. Hassel, L. Grönberg, P. Helistö, H. Seppä \\ VTT, P.O. Box 1000, FIN-02044 VTT, Finland
}

\begin{abstract}
Power generation and synchronisation in Josephson junction arrays have attracted attention for a long time. This stems from fundamental interest in nonlinear coupled systems as well as from potential in practical applications. In this paper we study the case of an array of junctions coupled to a distributed transmission line either driven by an external microwave or in a self-oscillating mode. We simplify the theoretical treatment in terms of harmonic analysis and power balance. We apply the model to explain the large operation margins of SNS- and SINIS-junction arrays. We show the validity of the approach by comparing with experiments and simulations with self-oscillating es-SIS junction arrays.
\end{abstract}

Josephson junctions (JJs) are well known to be able to generate microwave power. Their dynamics can also phaselock either to an external microwave bias or to the self-generated signal from an array of junctions [1]. A common configuration is to couple a number of JJs through a distributed transmission line resonator 22. Both overdamped 2] and underdamped [3] junctions in this configuration are known to be able to mutually synchronize. Long arrays of JJs coupled to a transmission line driven by external microwave bias are known to exhibit properties, which can only be explained by the collective dynamics of the JJ-transmission line system [4, 5].

Coupled Josephson junction arrays have been modelled by semiclassical dynamical simulations [7, 8, 9, 10], or in terms of lengthy perturbation analysis 11]. The dynamics of coupled arrays is complex making engineering of such devices challenging, often based on trial and error. However, in most practical situations it suffices to study the situation, where all the junctions are phase-locked to a driving signal (either external or self-generated). Recognizing this, we develop a simplified modelling technique. We have previously considered the case of external microwave bias, for which we calculated the gain in the limit of very small power self-generation [5]. Here we extend the analysis to the case of arbitrarily large gain, and apply it to self-stabilized overdamped JJ arrays. We analyze also the self-oscillating array by means of a power balance equation. Finally, we compare the results of the analytic model with simulations and experiments.

We assume that the tunnel element is effectively voltage biased. This is ensured by a small impednace $|Z(f)| \ll$ $\Phi_{0} f / I_{c}$, connected in parallel with the tunnel element (see Fig. 1(a)). Here $I_{c}$ is the critical current, $\Phi_{0}$ is the flux quantum and $f=\omega / 2 \pi$ is the drive frequency. We further assume that the dynamics is phase-locked to the driving signal. Below we drop the explicit $f$-dependence from the function notation, since all the quantities are evaluated at that frequency. The condition for phase-locking (to the first Shapiro step) is [ $]$

$$
\left|\delta I_{0}\right|<I_{c}\left|J_{1}\left(\widetilde{i}_{1}\right)\right|,
$$

where $\widetilde{i}_{1}=|Z| I_{1} / \Phi_{0} f, \delta I_{0}=\left(I_{b}-\Phi_{0} f / R\right)$ and $J_{1}$ is the Bessel function of the first kind. Here $I_{b}$ is the DC bias current, $R$ is the DC resistance in parallel to the junction and $I_{1}$ is the amplitude of the driving signal.

Using the assumptions above, we get the fundamental frequency component of the Josephson current $I_{J 1}$ (see Fig. 1(a)) 5]. Within harmonic approximation the voltage across the tunnel element is $V_{J 1}\left(I_{1}\right)=Z\left(I_{1}-I_{J 1}\left(I_{1}\right)\right)$, where the phase is referred to the driving signal $I_{1}$. We define dynamical impedance $Z_{d}\left(I_{1}\right)=R_{d}+i X_{d}=V_{J 1}\left(I_{1}\right) / I_{1}$, in series with $\omega L_{t} \gg X_{d}$, where $L_{t}$ is the transmission line inductance per junction length. We neglect $X_{d}$, assuming $X_{d}<\omega L_{t}$. The effects discussed here follow from the real part $R_{d}\left(I_{1}\right)=\operatorname{Re}\left(Z_{d}\left(I_{1}\right)\right)=\operatorname{Re}(Z)-\operatorname{Re}\left(Z I_{J 1} / I_{1}\right) . \operatorname{Re}(Z)$ is the real part of the linear impedance parallel to the junction, and $\operatorname{Re}\left(Z I_{J 1} / I_{1}\right)$ is due to the Josephson effect.

Knowing $R_{d}$, various properties of arrays are readily obtained. We consider first the case of external microwave drive (Fig. 1(b)). With $\left|R_{d}\right| \ll \omega L_{t}$ the (nonlinear) attenuation per junction is

$$
\eta\left(I_{1}\left(x_{k}\right)\right)=-\frac{1}{2} \frac{R_{d}\left(I_{1}\left(x_{k}\right)\right)}{Z_{0}},
$$

where $Z_{0}$ is the transmission line impedance. If $R_{d}>0$, the junction attenuates and if $R_{d}<0$, it amplifies the driving current. This is a generalization from the theory of low-loss transmission lines (e.g. 12]). The current distribution $I_{1}\left(x_{k}\right)$ along an array $\left(x_{k}\right.$ being the position of the $k$ :th junction) can be recursively obtained from $I_{1}\left(x_{k+1}\right)=I_{1}\left(x_{k}\right) \exp \left(\eta\left(I_{1}\left(x_{k}\right)\right)\right)$. This also generalizes our previous results [5] to arbitrarily large gains. 

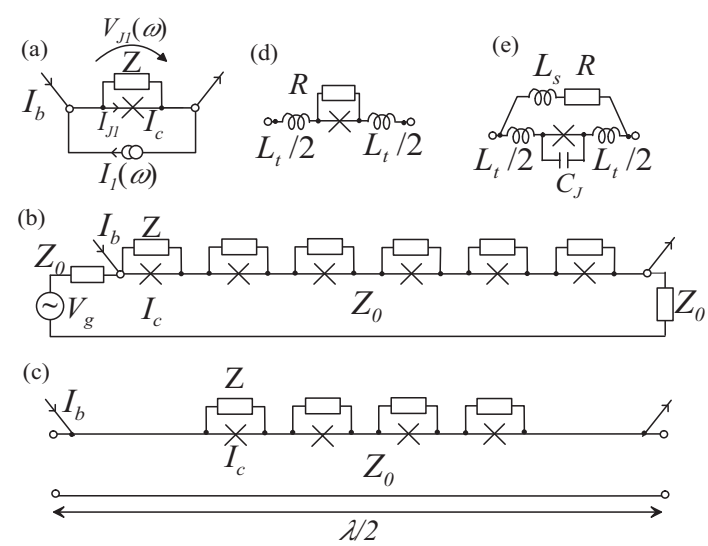

FIG. 1: (a) A microwave current biased junction. (b) A Josephson array coupled to a matched transmission line with external RF source. (c) A Josephson array coupled to a $\lambda / 2$-resonator. An $n \lambda / 2$ resonator is a straightforward generalization. (d) JJ with resistive environment. (e) JJ with capacitive environment.

Our second case is a resonator driven by self-generated power of the JJ:s (Fig. 1(c)). We note that the generated (or dissipated) power of a JJ at position $x_{k}$ is $-R_{d}\left(I_{1}\left(x_{k}\right)\right) I_{1}\left(x_{k}\right)^{2} / 2$. The current distribution in the system can therefore be solved by means of a power balance equation

$$
-\frac{1}{2} \sum_{k=1}^{N} R_{d}\left(I_{1}\left(x_{k}\right)\right) \times I_{1}\left(x_{k}\right)^{2}=P_{e x},
$$

where the left side is the generation due to all $N$ JJ:s and $P_{e x}$ is the excess loss, i.e. all power loss mechanisms not related to the junction or the shunt. In a high-Q resonator with $\left|R_{d}\right|,\left|X_{d}\right| \ll \omega L_{t}$ the resonant frequency determines the generated frequency. The position dependence of $I_{1}(x)$ depends also only on the resonator properties, whereas the overall amplitude depends on the gain and dissipation of JJ:s and on $P_{e x}$. The criterion (11) needs to be satisfied for all $I_{1}\left(x_{k}\right)$.

We identify two limits for $R_{d}$ in terms of junction type. The first is a resistive environment (Fig. 1(d)): $Z=R$ at the drive frequency. This is relevant to SNS or SINIS junctions with appropriate parameters. In this case we get, using above definition and results of Ref. [5] for $R_{d, R}=R_{d}(Z=R)$

$$
R_{d, R}\left(I_{1}\right)=R\left(1-2 \frac{I_{c} R}{\Phi_{0} f} \frac{J_{1}^{\prime}\left(\tilde{i_{1}}\right)}{\widetilde{i_{1}}} \sqrt{1-\left(\frac{\delta I_{0}}{I_{c}}\right)^{2} J_{1}^{-2}\left(\widetilde{i_{1}}\right)}\right),
$$

in which $J_{1}^{\prime}$ is the derivative of the Bessel function.

The second limit is a capacitive environment, $Z \approx 1 / j \omega C$, which is valid for unshunted SIS junctions. The subgap leakage resistance can typically be neglected. This is also a good approximation for externally shunted SIS (es-SIS) junctions, if $\left|R+\omega L_{s}\right| \gg 1 / \omega C$ (Fig. 1(e)). Here $L_{s}$ is the series inductance of the shunt resistor. We get

$$
R_{d, C}\left(I_{1}\right)=\operatorname{Re}(Z)-\frac{\Phi_{0} f}{2} \frac{\delta I_{0}}{I_{1}^{2}}
$$

where $\operatorname{Re}(Z) \approx 0$ for unshunted junctions. For es-SIS junctions the losses in the shunts can be the dominant mechanism of linear dissipation, although their effect on JJ oscillation (second term in (15)) is small. For the arrangement of Fig. $1(\mathrm{e}), \operatorname{Re}(Z) \approx\left(L_{e f f} / L_{s}\right)^{2} R$ if $L_{s} \gg L_{e f f}$, where $L_{e f f}=L_{t}-1 / \omega^{2} C$.

It is experimentally known that long overdamped arrays driven by external microwave compensate for the transmission line attenuation [4]. This is the reason why SNS and SINIS Josephson voltage arrays work in spite of the huge attenuation due to intrinsic damping [4, 13]. With the above notation, self-compensation occurs if the amplitude of the propagating signal is initially between $I_{1, \min }$ and $I_{1, \max }$ such that (see (2), (4))

$$
\begin{aligned}
& \eta\left(I_{1}\right)=0, I_{1}=I_{1}^{\prime}, \\
& \eta\left(I_{1}\right)>0, I_{1, \text { min }}<I_{1}<I_{1}^{\prime}, \\
& \eta\left(I_{1}\right)<0, I_{1}^{\prime}<I_{1}<I_{1, \text { max }} .
\end{aligned}
$$




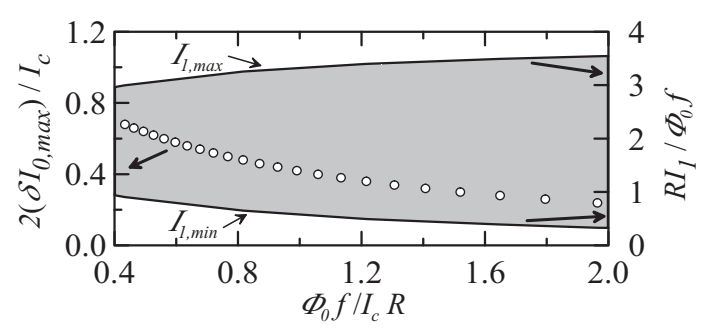

FIG. 2: Operating margins of an overdamped JJ array in self-stabilizing mode according to (11) and (6). Open circles (left axis): Maximum step width as a function of $\Phi_{0} f / I_{c} R$. Gray-shaded area (right axis): pump current range maximising the step width.

In addition, $I_{1, \min }$ and $I_{1, \max }$ need to satisfy (1). Then $I_{1}(x)$ approaches the stable point $I_{1}^{\prime}$ towards the end of the transmission line. Fig. 2 shows the maximum step width $2 \delta I_{0, \max }$ and pump current margin $I_{1, \max }-I_{1, \min }$, within which the array self-stabilizes the microwave current. Typical values of the design parameter $\Phi_{0} f / I_{c} R$ of overdamped arrays vary between 0.5 and 2 . In this range, the maximum self-stabilized step width varies between $0.2 I_{c}$ and $0.6 I_{c}$. Using the parameters of Fig. 2 in [4] $\left(\Phi_{0} f / I_{c} R \simeq 1.3\right)$, our model predicts $2 \delta I_{0, \max } \approx 400 \mu \mathrm{A}$ and $20 \log _{10}\left(I_{1, \max } / I_{1, \min }\right) \approx 18 \mathrm{~dB}$, which are in excellent agreement with the exprimental data $\left(2 \delta I_{0, \max } \approx 400 \mu \mathrm{A}\right.$ and $\left.20 \log _{10}\left(I_{1, \max } / I_{1, \min }\right) \gtrsim 10 \mathrm{~dB}\right)$.

In SIS and es-SIS junctions, $\eta$ does not change sign as function of $I_{1}$ (see the second term in (15)). Therefore the tendency for power self-stabilization is not present. The much smaller attenuation of the capacitive environment makes phase-locking of all junctions possible in this case for a reasonable number of junctions [5, 14].

Finally, to study self-oscillating arrays, we prepared arrays embedded in open-ended $\lambda / 2$ or $\lambda$ microstrip resonators. The circuits were fabricated using the $\mathrm{Nb} / \mathrm{Al} / \mathrm{AlOx} / \mathrm{Nb}$ fabrication line of VTT [15] and measured at $4.2 \mathrm{~K}$. The resonators were $800 \mu \mathrm{m}$ or $1600 \mu \mathrm{m}$ long $\mathrm{Nb}$ strips having a resonant frequency $f$ nominally about $80 \mathrm{GHz}$. The resonators had 2-28 es-SIS JJ:s centered around the node(s) of the standing wave. A photograph of one of the structures is shown in Fig. 3(a). Measured IV curves are shown in Fig. 3(b).

The AC current distribution in the resonators is given as $I_{1}(x)=I_{0} \sin (2 \pi x / \lambda)$. The excess loss is given as $P_{e x}=\omega L_{T 0} I_{0}^{2} / 2 Q$. where $L_{T 0}$ is the total inductance of the resonator and $Q$ is the intrinsic Q-value. Using Eqs. (3) (15) the power balance stands $\frac{1}{2} \sum_{k=1}^{N}\left(\left(L_{e f f} / L_{s}\right)^{2} R \times\left(I_{0} \sin \left(2 \pi x_{k} / \lambda\right)\right)^{2}-2 \Phi_{0} f \times \delta I_{0}\right)=\pi f L_{t 0} I_{0}^{2} / Q$. This is solved for $I_{0}$. For $\delta I_{0}<0$, no physical solution exist, since $R_{d, C}$ can be negative only for positive $\delta I_{0}$. We get

$$
\begin{aligned}
I_{1}(x) & =\sqrt{\frac{N \Phi_{0} f \times \delta I_{0}}{2 \pi f L_{t 0} / 2 Q+(1 / 2)\left(L_{e f f} / L_{s}\right)^{2} R \sum_{k=1}^{N} \sin ^{2}\left(2 \pi x_{k} / \lambda\right)}} \sin \left(\frac{2 \pi x}{\lambda}\right) . \\
\delta I_{0} & <I_{c}\left|J_{1}\left(\frac{I_{1}\left(x_{k}\right)}{2 \pi \Phi_{0} f^{2} C}\right)\right| .
\end{aligned}
$$

Here we have rewritten (11) for convenience. For the resistive environment also the negative side of the step $\delta I_{0}<0$ appears.

The solutions for which all junctions are phase-locked to the driving signal appear in the IV curves of Fig. 3 as steps with voltage $\langle V\rangle=N \Phi_{0} f$ (for simplicity we omit the small dependence of $f$ on the bias point due to $X_{d}$ ). This happens for the solutions of (17) that satisfy (8) for all $x_{k}$. Solutions where no phase locking occurs are described by a resistive IV curve determined by the shunt resistors. We assume here that these are the only types of solutions.

The theoretical IV curves obtained in this way are shown as solid lines in Fig. 3(b). The fitting parameters are the Q-value and the resonant frequency $f$. The Q-value was fitted to the case with $N=2$, for which the effect of the parameter spread is the smallest. The result $Q=420$ was used for all the arrays. This corresponds to a transmission line loss of $\alpha=\pi / \lambda Q \times 8.7 \mathrm{~dB} \approx 41 \mathrm{~dB} / \mathrm{m}$, very close to that of an earlier measurement $(50 \mathrm{~dB} / \mathrm{m})[14]$. The loss is dominated by the dielectric $\left(\mathrm{PECVD} \mathrm{SiO}_{2}\right)$ loss. The resonance frequencies for all devices were very close to the nominal value $80 \mathrm{GHz}$. Other parameters were obtained independently either from the IV curves or from the known circuit and material properties.

The fact that steps appear only for $\delta I_{0} \gtrsim 0$ as expected is clarified for $N=2$ in Fig. 3(b). The step widths are close to the values predicted by the theory, though smaller. The difference is explained by parameter spread (the shunt resistances varied up to $\pm 5 \%$ within a chip, changing the effective bias point $\delta I_{0}$ by tens of $\mu \mathrm{A}$ ). 


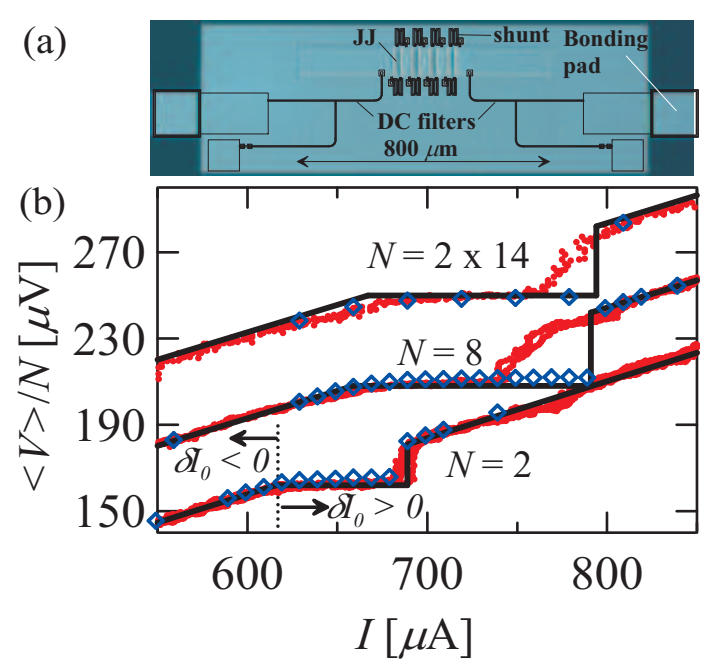

FIG. 3: (a) An experimental array with a $\lambda / 2$-resonator having $N=8 \mathrm{JJ}$ :s. Typical circuit parameters are $I_{c}=280 \mu \mathrm{A}$, $C=59 \mathrm{pF}, R=0.26 \Omega, L_{s}=3.4 \mathrm{pH}, L_{t}=0.2 \mathrm{pH}, L_{t 0}=5.7 \mathrm{pH}$ and $f=80 \mathrm{GHz}$. (b) Current-voltage curves measured from the resonators with $N$ JJs (small red circles). The device with $N=2 \times 14 \mathrm{JJ}$ :s is based on a $\lambda$-resonator. Others are based on $\lambda / 2$ resonators. The voltage is scaled to that of a single junction and curves are lifted by $40 \mu \mathrm{V}$ with respect to the one below for clarity. The analytic curves are shown by solid black lines and the simulated points are denoted by large blue diamonds.

To further study the validity of the model we performed time-domain simulations using Stewart-McCumber model for the junctions and a distributed model of a dissipative transmission line [12] to describe the resonator sections. We found good agreement with the analytic model. The model step widths are well reproduced, though the small bending of the plateau due to finite $X_{d}$ is not accounted for by the single-frequency model. As assumed, in all cases the numerical solutions were such that either all the junctions were synchronized or nonsynchronized. However, for simulated devices having even larger number of JJs per resonant node than our experimental devices, we found also partially synchronized states. Also in these cases the bias range of the completely synchronized state was correctly predicted by the analytic model.

In summary, we have developed a simple analytic technique for quantitative modelling of distributed JJ systems with a strong preferred frequency set by either an external RF signal or a high-Q resonance. The results clarify differences between underdamped and overdamped systems, a source of some controversy recently. The model provides an intuitive way of understanding the dynamics of phase locking and power generation in JJ arrays. The model predicts results in agreement with simulations and experiments and it can be used to engineer practical devices, such as mm-wave local oscillators and JJ voltage standards. An interesting alternative voltage standards is a JJ array with no external microwave source, locking the self-generated signal directly to a frequency reference. The power balance equation is easily generalized to externally loaded systems to calculate the power output. Other types of physical systems can be analyzed similarly. For example, the exact dual for systems described here is a distributed parallel array of quantum phase slip junctions [16], where the "conductor gain" of JJ arrays is replaced by a "dielectric gain".

The work was partially supported by MIKES (Centre for Metrology and Accreditation), Academy of Finland (Centre of Excellence in Low Temperature Quantum Phenomena and Devices) and EU through RSFQubit (FP6-3749). The authors wish to thank Antti Manninen for useful discussions.

[1] A. K. Jain, K. K. Likahrev, J. E. Lukens, and J. E. Sauvageau, Phys. Rep. 109, 310 (1984).

[2] K. Wan, A.K. Jain, and J.E. Lukens, Appl. Phys. Lett. 54, 1805 (1989).

[3] P. Barbara, A. B. Cawthorne, S. V. Shitov, and C.J. Lobb, Phys. Rev. Lett. 82, 1963 (1999).

[4] H. Schulze, R. Behr, F. Mueller, and J. Niemeyer, Appl. Phys. Lett. 73, 996, 1998.

[5] J. Hassel, P. Helistö, L. Grönberg, H. Seppä, J. Nissilä and A. Kemppinen, IEEE Trans. Instrum. Meas. 54, 632 (2005).

[6] R. L. Kauz, and R. Monaco, J. Appl. Phys. 57, 875 (1985).

[7] A. B. Cawthorne, P. Barbara, S. V. Shitov, C. J. Lobb, K. Wiesenfield, and A. Zangwill, Phys. Rev. B 60 (1999).

[8] E. Almaas, and D. Stroud, Phys. Rev. B. 65, 134502 (2002).

[9] G. Filatrella, N. F. Pedersen, C. J. Lobb, and P. Barbara, Eur. Phys. J. B 34, 3 (2003). 
[10] K.-T. Kim, M.-S.-Kim, Y. Chong, and J. Niemeyer, Appl. Phys. Lett. 88, 062501 (2006).

[11] D. Tsygankov, and K. Wiesenfeld, Phys. Rev. E 66, 036215 (2002).

[12] D. M. Pozar, "Microwave Engineering", 2nd. ed., John Wiley \& Sons, inc. (1988).

[13] C. A. Hamilton, C. J. Burroughs, S. P. Benz, and J. R. Kinard, IEEE Trans. Instrum. Meas. 46, 224 (1997).

[14] J. Hassel, H. Seppä, L. Grönberg, and I. Suni, Rev. Sci. Instrum. 74, 3510 (2003).

[15] L. Grönberg, J. Hassel, P. Helistö, M. Kiviranta, H. Seppä, M. Kulawski, T. Riekkinen, and M. Ylilammi, Extended abstracts of International Superconductivity Conference 2005, Noordwijkerhout, The Netherlands, O-W.04 (2005).

[16] J. E. Mooij, and Yu. V. Nazarov, Nature Physics 2, 169-172 (2006). 